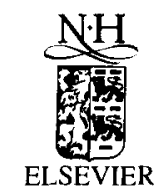

ELSEVIER

\title{
Formulations in first encounters
}

\author{
Tony Hak*, Fijgje de Boer \\ Department of Sociology, M5-10, Erasmus University, P.O. Box 1738, NL-3000 DR Rotterdam. \\ The Netherlands
}

Received 10 August 1993; revised version 14 July 1994

\begin{abstract}
The paper describes and compares the use and function of the formulation-decision pair in three types of diagnostic interviewing. The investigatory type of interviewing, which typically occurs in the medical interview, is characterized by the absence of formulations. In the exploratory type of interviewing, which typically occurs in the psychiatric interview, the interviewer uses the formulation-decision pair for exploring the patient's experiences. He does not, however, formulate his professional assessment in the interview. In the collaborative type, which typically is found in psychotherapy, the formulation-decision pair is the main interactional device used by the interviewer for translating the patient's troubles into a professional problem definition. It is suggested that the patient's protoprofessionalization, which is typical for psychotherapy, already begins in the initial interview and that it is an effect of the interviewer's specific use of the formulation-decision device in the interview.
\end{abstract}

\section{Introduction}

In her seminal study 'Problem (re)formulation in psychotherapy', Davis concludes that formulations constitute an important, if not the main conversational activity of the therapist during the initial interview (1984: 43). In a detailed and subtle analysis, she describes the way in which the therapist makes use of formulations in order to transform the client's initial set of troubles into a professional problem, i.e. a problem that is treatable in the context of psychotherapy. ${ }^{1}$ In the diagnostic process in first encounters in general practice and in psychiatry, formulations do not play such a role, though for different reasons. Typically, in the medical interview, which is characterized by abrupt topic shifts, there are no formulations of the patient's talk. Formulations can be found in the diagnostic interview in psychiatry, but these appear to have quite another function than in the initial interview in psychotherapy.

* Corresponding author. E-mail: hak@risbo.fsw.eur.nl

1 For the process of reformulating client's problems into a professional problem definition, see also Weingarten (1990), who distinguishes between different types of reformulation. 
The aim of this paper is, first, to describe and compare in a more detailed way the use and function of the formulation-decision pair in three types of interview, the interrogatory type (which is characteristic for the medical interview), the investigatory type (which can be found mainly in psychiatric diagnostic interviews), and the collaborative type (which is characteristic for the initial interview in psychotherapy). Next, the implications will be discussed of the conversational properties of the formulation-decision pair (described by Heritage and Watson, 1979) for the position of the client in psychotherapy. First, we will summarize Heritage and Watson's analysis of the properties of the formulation-decision pair.

\section{The formulation-decision pair in conversation analysis}

Heritage and Watson start from the observation, furnished by Garfinkel and Sacks, that a member may treat some part of the conversation

"as an occasion to describe that conversation, or explain it, or characterize it, or explicate, or translate,
or summarize, or furnish the gist of it, [...]. A member may use some part of the conversation as an occa-
sion to formulate the conversation." (Garfinkel and Sacks, 1970: 350)

In many cases, this 'saying-in-so-many-words-what-we-are-doing' is achieved by producing a paraphrase of some prior utterance, preserving relevant features of the prior utterance while also recasting it. These formulations thus manifest three central properties: preservation, deletion and transformation. (We will present examples below.) Typically, formulation is done by a 'news recipient' (e.g. a therapist), showing in this way his understanding of the news delivered by a 'news deliverer' (e.g. a client presenting his problems). See, for instance, the following extract from a faceto-face interview with the 'Slimmer of the Year', relayed on radio (Extract 1).

Extract 1 (from Heritage and Watson, 1979: 132)

(I=interviewer; $S=S$ limmer of the Year; $F=$ formulation; $D=$ decision)

SI When I was at college I think I looked like a matronly fifty. And I was completely alone one weekend and I got to this stage where I almost jumped in the river. I just felt life wasn't worth it any more - it hadn't anything to offer and if this was living I had had enough.

F I1 You really were prepared to commit suicide because you were a big fatty.

D S2 Yes, because I - I just didn't see anything in life that I had to look forward to.

In this extract, the interviewer shows his understanding of the slimmer's talk by formulating it. Heritage and Watson make a distinction between two types of conversational formulation: gists and upshots. Gists primarily constitute clarifications, or demonstrations of comprehension or in-touchness with the talk thus far. The interviewer's formulation in Extract 1 is an example of a gist. Upshots presuppose some unexplicated version of gist. Extract 2 presents an example of an upshot (in I2). 
Extract 2 (from Heritage and Watson, 1979: 134)

(I=interviewer; $\mathrm{R}=$ respondent)

Il If occasion -if occasion "rises again will you take similar action?

RI Well we have never hesitated so far to er take action where er freedom is being abused.

F I2 So there might be another occasion on which you will use the law against unions.

D R2 Not necessarily against unions but against any body or which has become over mighty er and is abusing its responsibilities.

In the process of the news recipient's formulation, some 'candidate reading' is offered for a preceding stretch of talk, whose adequacy or preferredness may subsequently be decided upon by the news deliverer (Heritage and Watson, 1979: 138). Thus, formulation and decision constitute an adjacency pair, which means that the absence of a news deliverer's decision (confirmation or disconfirmation) following a news recipient's formulation is hearable and must be accounted for (ibid.: 142).

An important finding, reported by Heritage and Watson (1979: 143; see also Pomerantz, 1984), is that confirmations are massively preferred. This is because disconfirmations may jeopardize the sense of 'the talk so far' as an accountable test which is available as an unequivocal resource for the ongoing members' collaborative constructions. The direct faulting of a formulation may even imply a challenge to the formulator's capacity and competence in monitoring the gists of talk. Conversely, inadequate responses to formulations may stimulate an inspection of that inadequacy's motivation (1979: 144). The formulation-decision pair, then, is a builtin part of rendering conversations preservable and reportable, and it is in this sense that formulations may be said to 'fix' what will have turned out to be a (the) topic.

After this very short introduction to some properties of the formulation-decision pair as a conversational device, we will now turn to the question what the use and function of the formulation-decision pair are in first encounters.

\section{The interrogatory type of interviewing}

In his analysis of the medical encounter, Mishler (1984) describes how a patient's talk often is interrupted abruptly by the physician, who asks for more detailed and technical information about the complaints. This can be illustrated by the following excerpt from Mishler's data (Extract 3).

Extract $3^{2}$ (from Mishler, 1984: 65)

( $\mathrm{D}=$ physician; $\mathrm{P}=$ patient)

DI What's the problem.

2 The following symbols have been used in the transcripts:

(0.5) pause of 0.5 seconds

(word) word(s) unclear but 'retrieved' as far as possible by transcriber 
Pl ((chair noise)) had since. last Monday evening so it's a week of sore throat

D2 hm

P2 which turned into a cold (2.0) and then a cough.

D3 A cold you mean what? Stuffy nose?

P3 uh Snuffy nose yeah not a chest $(0.5)$ cold.

D4 And a cough.

P4 And a cough (0.2) which is the most irritating aspect.

D5 Okay. Any fever?

P5 (0.6) Not that I know of. (0.4) I took it a couple of times in the beginning but haven't felt like-

D6 How about your ears?

P6 (1.5) Before anything happened (0.4) I thought that my ears (0.6) might have felt a little bit funny but $(0.4)$ I haven't got any problem(s).

D7 Okay. (0.8) Now this uh cough what are you producing anything or is it a dry cough?

This extract shows the beginning of a medical encounter. The difference in discourses that are being used by patient and physician can easily be recognized. The patient has to answer the questions and to give the necessary information in order for the physician to make up a diagnosis. The physician guides the patient's talk by requesting medical technical information and by acknowledging that he or she has received enough information.

This type of conversation can be characterized as a series of exchanges that are built up according to a three-part structure. This structure consists of:

(a) a request from the physician;

(b) a response from the patient;

(c) a post-response assessment by the physician (e.g. "Okay"), to which a new request is added, indicating the beginning of the next cycle (topic shift).

Sometimes questions of clarification (e.g. D3 and D4) are posed, but these do not go beyond the technical information that the physician apparently needs. Apart from the many pauses in patient's talk, there is no indication that patient and physician have any trouble in understanding each other or that the conversation was perceived of as odd on the part of the patient. Although this may be taken as an indication of the unproblematic nature of the conversation in this excerpt, it is remarkable that we do not see the physician formulating the patient's talk. If we take ordinary conversation as a reference point, the absence of formulation may be interpreted as a hearable disinterest on the physician's part in the collaborative establishment of common comprehension of the talk thus far. In other words, the absence of formulations makes

\footnotetext{
( ) utterance produced but its sense could not be discerned

((sobbing)) transcriber's comments

nine underlining a word indicates conversationalist's emphasis on that word or part of word [...] data omitted

We have reduced Mishler's detailed transcript (particularly by eliminating indications of overlap, intake of breath, and the like), because this amount of detail is not necessary for our purpose at hand.
} 
apparent the physician's implicit view that he, and only he, is in the position to decide on the comprehensibility and the relevance of the ongoing talk.

Mishler (who studied 25 taped medical encounters) contends that this three-part utterance sequence is a "regular and routine occurrence in the talk between patients and physicians" (1984: 68). Although it may be a regular occurrence, it is certainly not the only type of utterance sequence occurring in the medical encounter. Extract 4 , for instance, shows a different structure.

Extract 4 (from Mishler, 1984: 129)

( $\mathrm{D}=$ physician; $\mathrm{P}=$ patient $)$

D1 I'm Doctor Gerson.

P1 I know.

D2 Okay. (1.0) Now let's see. You were referred here, actually they sent you up here from medical clinic (1.0) from the screening clinic rather.

P2 Yeah. Well I was sent up here from uh- from neurology really (1.0) because I told them what my symptoms were (1.0) and uh they said okay (0.6) we'll get you up there. But first I went to see my doctor. He put me on a diet. He gave me some pills (0.6) and he said while you're in there go. (1.2) In other words don't come back to me (1.0) until you've had X-rays taken. (1.0) That's what he implied.

D3 (1.0) Okay. Now you've had according to this thing (3.5) you haven- you had an ulcer at age nine?

P3 Um about- between nine- nine and eleven I had the first one.

D4 The first one?

P4 And then- uh the two years later I developed a second one.

D5 (0.4) That was about thirteen or so.

P5 Between- between nine- nine and thirteen. (0.8) The only thing-

D6 That's when you had your second one.

P6 Yes. The only thing I can remember is that my doctor was shocked to death because he never knew a girl my- my age that had two ulcers.

D7 And how did- how did the ulcers present. What uh- what happened? (0.6) Just pain or?

In this extract, the patient's utterance "The only thing I can remember is that my doctor was shocked to death because he never knew a girl my- my age that had two ulcers" (P5-P6) seems to be unwelcome. One indication of this is that the patient's talk, her turn, is interrupted (P5/D6), and that it subsequently is totally dismissed by means of an abrupt topic change (P6/D7).

In Extract 4, the physician makes use of the formulation-decision pair in D5 and D6. Here, the physician formulates the gist of the patient's utterance in P4 by paraphrasing it. The paraphrase preserves a part of it ('the second one') and transforms 'two years later' into 'about thirteen or so'. This is confirmed by the patient in P6 ("Yes"), although this is qualified immediately: "The only thing I can remember is ...". It is clear that the formulation has the very restricted function of only clarifying one utterance (P4). There is, however, in this extract no instance where a longer 
stretch of talk is formulated or where the physician formulates his assessment of what he has heard.

It is typical for the interview style described by Mishler that the physician shifts topic abruptly, without closing the prior topic by means of formulation. Mishler notes that this is not seen as something unfamiliar by the patient:

"As members of this culture we, as observers, and the patient, are likely to assume that the physician has 'reasons' for his questions. It is somewhat remarkable, but understandable on the basis of this shared assumption, that the patient does not reject any of his questions as inappropriate, however disjunctive they are with previous content, but makes an effort to answer them. Presumably the physician's questions reflect the biomedical model [...]. Nonetheless, the model is not made explicit to the patient. It is invisible and inaudible in the discourse." (Mishler, 1984: 120)

At least, that seems to be the 'normal' case. The 'good' patient does not expect to be considered a competent party to whom it ought to be explained what the order of the talk is. He normally accepts that the physician imposes his order, even if this renders the conversation opaque to him. Thus, the patient's assumption of a logical order in the physician's questioning (although this order is invisible and inaudible in the physician's talk itself) and the patient's effort to be cooperative are the main guarantees for a more or less smooth progress of the encounter.

Because of its conversational asymmetry we call the type of interviewing as described by Mishler, interrogatory. For examples of two other types of interviewing, exploratory and collaborative, we will now turn to examples from our own research.

\section{The exploratory type of interviewing}

Extract 5 is an excerpt from a psychiatric diagnostic interview.

Extract $5^{3}$

( $\mathrm{N}=$ social psychiatric nurse; $\mathrm{P}=$ patient)

P1 I've been used all my life. ((pause, then very softly:))

(what I had already ( ) the whole country)

\footnotetext{
3 Extracts 5 through 10 are translations of Dutch data. The social psychiatric nurse in Extract 5 is working in the Department of Emergency Psychiatry of a 'Regional Institute for Ambulatory Mental Health Care' (Dutch acronym RIAGG). The patient is a woman being visited at home by the nurse. The day before, the woman had been seen by another social psychiatric nurse, who could not, however, assess her symptoms clearly at that time. At the time of the present interview, this nurse is visiting the patient in order to decide whether admission into a mental hospital is necessary. During this home visit, the nurse advises the patient to go to an emergency center. Later during that day, the nurse visits her again at the emergency center and informs his colleague at the center about the case. All these conversations were taped and transcribed. This extract is part of the transcript of the conversation at the patient's parents' home.

This data has been collected in the framework of a research project on decision-making in emergency psychiatry, in which 15 encounters between social psychiatric nurses and patients were taped, transcribed and analyzed. For further analyses of this data, see De Boer and Hak (1986), Hak (1989), and Hak (1992).
} 
N1 Sorry.

P2 The whole country knew that.

N2 What did the whole country know?

P3 What I just said.

N3 I don't understand. I just don't get it.

P4 I've just been used all my life.

N4 By whom?

P5 By boys.

N5 Yes. And how does the whole country know about this?

P6 It was broadcast.

N6 It was broadcast. On radio or something?

P7 And on TV.

N7 That you're being used?

P8 No uh with whom I went to bed.

F N8 Strange.

D P9 Yes, I too consider it rather strange.

What is remarkable in this piece of talk on everyday life, in comparison to Extracts 3 and 4 , is the absence of an abrupt topic shift. Instead, the nurse requests the patient to expand upon her story, which is explicitly biographical in nature. At every turn, the nurse requests more information on the "patient's contextually-grounded experiences of events and problems in her life" (Mishler, 1984: 104; see above). His questions are not disjunctive with what was previously said, but rather are attempts at clarification in order to find out what the patient wants to tell him. Detailed and trivial information about everyday life is not dismissed in this context.

It is the nurse who does the conversational work of connecting pieces of talk together into an understandable whole, not the patient, as in Extracts 3 and 4. There is no formulation in the extract, but the nurse accounts for this absence by explaining his difficulty in understanding. His assessment of the whole stretch of talk as 'strange' (in N8) can be considered as substituting for a formulation, and evokes an appropriate confirmation (in P9).

At first sight, however, it may seem arbitrary to compare this extract with Extracts 3 and 4 , because it is not evident that the nurse is doing something comparable to what the physician is doing in those extracts. There is, however, evidence in the nurse's reports about this patient that in Extract 5, the nurse is doing exactly the same as the physician is doing in Extracts 3 and 4, namely collecting symptoms in order to construct a diagnosis. An example is the following assessment of Extract 5, as reported by the nurse to a colleague: "Her sexual life, things that happened in it, appears to her as if they were broadcast on the radio, or at least that somehow happened. So in that sense she has quite clear circumscribed delusional ideas".

Thus, the nurse is not simply talking to the patient about her experiences as an interested companion, but he is at the same time assessing her talk as evidence of delusions. Just as in the interrogatory type of interviewing, the professional model is not made manifest to the patient, for whom it is invisible and inaudible. A difference, however, is that the patient's biographical information is not dismissed. 
Instead, it is expanded upon and subsequently used as 'raw material' for the construction of a psychiatric diagnosis.

A provisional conclusion is that in the investigatory type of interviewing, the formulation-decision pair may be used precisely in order to do what it is supposed to do, namely "to describe that conversation, or explain it, or characterize it, or explicate, or translate, or summarize, or furnish the gist of it" (Garfinkel and Sacks, 1970: 350). The formulation-decision pair, i.e. formulating the news received and exposing it to the news deliverer's assessment, is an appropriate means to achieve just what the interviewer is aiming at, namely a maximally thorough exploration of the patient's life. However, the interviewer does not formulate the professional upshot of patient's talk, at least not in the encounter itself. It is formulated elsewhere, namely in the professional environment. In the context of this paper's focus on the formulation-decision pair, the pair's most important feature is that the professional assessment cannot be followed by a decision on the part of the patient, because the interviewer does not formulate the assessment in the interview itself. Thus, the exploratory type of interviewing marks a very restricted interest in the patient's talk. It is conceived of as merely a way of getting the patient to deliver symptoms.

Listening to the patient's stories about everyday experiences does not automatically mean that the patient's talk is taken for granted by the professional. On the contrary, the interviewer can express his feelings of non-comprehension (e.g. by saying "Strange", see N8 in Extract 5). He may even ironize over the patient's story, as is shown in Extract 6.

Extract $6^{4}$

( $\mathrm{N}=$ social psychiatric nurse; $\mathrm{P}=$ patient $)$

P1 Well, I find it rather unpleasant to uh well to go uh to go to sleep in my own room.

N1 Why? What is wrong with that room?

P2 This traffic, it is going on the whole night through.

N2 Mmmm.

P3 It bothers me. And uh in the morning at six o'clock the birds start whistling and uh that troubles me terribly. Because then I know that I cannot uh rest in a normal way.

N3 Yeah, yeah.

P4 It is irritating to me.

N4 Yes, they deprived you of your rest.

P5 Yes.

N5 And in the psychiatric hospital?

P6 And this is this is terribly annoying. I have nothing against birds but I mean in the way it is I mean it awfully annoys me.

\footnotetext{
4 This is another social psychiatric nurse, working at the same Department of Emergency Psychiatry. The patient is a man being visited by the nurse in the living room of a sheltered home, where the man now lives after having stayed, off and on, in a psychiatric hospital for some years. The patient requested to be admitted into a psychiatric hospital again. For more information on this data, see De Boer and Hak (1986).
} 
[data omitted]

I absolutely do not want to stay here.

F N6 And the birds are anywhere. You will find them particularly everywhere in the countryside.

D P7 Hihi yeah that is true. Yeah.

N7 Isn't it?

D P8 Yes, that's right.

N8 Even more than here I guess.

D P9 That's right yes.

N9 Than in the city.

P10 But uh I mean this pain in my head I do not know what it is.

The patient's talk in this excerpt was transformed into the professional assessment (in the subsequent report by the nurse; see Hak and De Boer, 1995) that "he pretends that he is not well". In the conversation, however, the nurse does not present this assessment explicitly. He does not dispute that the patient is not well, but only denies that there will be any use in going to a mental hospital for relief (in N6 and N8). Implicitly, this denial presupposes the nurse's understanding that birds annoy the patient, and that this is his reason for his soliciting admission to a mental hospital; in other words, what is presupposed is the nurse's understanding of the gist of the patient's talk. By explicitly eliciting the patient's confirmation of his opinion that leaving his present home will not solve the problem of the annoying birds (in N7), the nurse at the same time elicits a confirmation of his implicit understanding. The fact that the patient changes the topic (in P10) after having confirmed the nurse's opinion twice (in P7 and P9), implies that he does not challenge the implicit upshot of the talk so far, as presented by the nurse.

From these two examples of exploratory interviewing, we may conclude that the function of the formulation-decision pair, as used in the psychiatric interview, is to create a shared understanding of 'facts' in the patient's life - an understanding that can be subsequently transformed into a professional assessment. Because the exploratory type of interviewing depends upon an exploration of the patient's life, formulations (or similar conversational devices) must be used in order to check whether the patient's talk has been understood properly. The patient will experience the encounter as a 'proper' conversation, not fragmented, as in the case of interrogatory interviewing. The interviewer avoids carefully, however, to formulate his professional upshot of the patient's talk. This constitutes the main difference between the exploratory type of interviewing and the collaborative type, to be discussed in the next section.

\section{The collaborative type of interviewing}

Let us now look at the third type of interviewing, which we will call the collaborative type. In this type, the interviewer enters the patient's life just as in exploratory 
interviewing, but with another aim and, consequently, with a different result. Extract 7 , an excerpt from an initial interview in psychotherapy, illustrates this.

\section{Extract $7^{5}$}

( $\mathrm{C}=$ counsellor; $\mathrm{P}=$ patient $)$

P1 Well, the problem is, things come up, and that started, uhh, with my work, and that was really the main reason, in principle. [...] And the main stumbling block, I keep saying, that, for example, the, uhh, I can't say no. I am afraid that I uhh am not functioning well, at home, at my work, everywhere. I want to do everything well. [...] And last week I had the nerve to throw out everything to the managing director.

C1 Yes.

P2 And apparently he was startled by it and also startled by the absenteeism in our department.

C2 Yes.

[data omitted]

P3 And I now already feel relieved from a very big burden, because I have had this interview with him.

C3 Yes.

P4 For there was, uhh, that, I, for let me state it this way, that was in my eyes the most important point.

F C4 Yes, so really a lot of tension got off your back, because you started to ventilate, to disclose what is going on.

D P5 Yes, but that's happening very often to me, when I quarrel with my wife, that does not happen every day, but once in a while something comes up that I just blurt out.

[data omitted]

F C5 But does that mean, John, that somehow you are an introvert?

D P6 Yes, one hundred percent. It keeps simmering in my stomach.

F C6 It all stays a little bit in your mind and at a certain moment you spit out everything at once?

D P7 Yes, I am an introvert, yes.

In this extract, the interviewer first formulates the gist of the patient's utterances by paraphrasing it. The patient's "I now already feel relieved from a very big burden" (P3) is transformed into "so really a lot of tension got off your back" (C4). After the patient's confirmation (in P5), the nurse then formulates the upshot of the patient's talk (in C5: "you are an introvert"). The interviewer articulates a relationship between the label 'introvert' and the patient's talk in two ways: first (in C5) by

\footnotetext{
5 The counsellor is working in a RIAGG, and the client is a man referred by a general practitioner. The initial interview has been recorded. The report that the counsellor wrote after the interview also belongs to the research data. This data forms part of a research project, carried out by De Boer, entitled 'Sex differences in the construction of mental health care problems', in which data on the intake of 60 clients in eight RIAGGs has been collected and transcribed.
} 
explicitly presenting his formulation as the 'meaning' of the patient's talk, and second (in C6) by 'unpacking' his gloss ('introvert') by means of a paraphrase of what the patient just said. The interviewer's formulation "It all stays a little bit in your mind and at a certain moment you spit out everything at once?" paraphrases "It keeps simmering in my stomach" (P6) and "Something comes up that I just blurt out" (P5). The patient's repeated confirmation (P7) is the concluding move of this fine example of the collaborative construction of a label ('introvert') that can be used in the subsequent process of elaborating a professional problem definition.

The following extract is another example of collaborative interviewing in the initial interview:

\section{Extract $8^{6}$}

( $\mathrm{C}=$ counsellor; $\mathrm{P}=$ patient $)$

$\mathrm{C} 1$ ((Reads loudly from the general practitioner's referral letter. Then:)) What does this mean for you?

P1 Yes, I simply have a lot of difficulties with, things for myself uh, yes, with really defending myself.

[data omitted]

F C2 You appear not to be able to draw a line somewhere, huh, to, you know, it's up til here and not further. Do you recognize this within yourself, that you, when people are requesting something from you, even if you don't know it any more, that you nevertheless will do it?

D P2 Yes, I recognize that very clearly.

C3 Hn.

P3 I can't say no.

[data omitted]

F C4 And this, this is a typical thing you know, this difficulty with defending yourself, with not daring to say no, so really you could say, you are subassertive. If you understand its purport?

D P4 Yes.

In this extract, first, the interviewer formulates the gist of the patient's utterance (C1) by paraphrasing it as "not able to draw a line somewhere" and elicits a decision (in $\mathrm{C} 2$ ). Subsequently, after the patient's confirmation (in P2/P3), he formulates the professional upshot of the patient's talk (in C4): "you are subassertive". This last instance, in which a relationship is articulated between the patient's talk ("not daring to say no", which takes up the patient's "I can't say no") and the professional problem formulation, is an example of how a professional definition of a patient's problem can be constructed by formulating the patient's talk. ${ }^{7}$

\footnotetext{
6 The client is a woman referred by a general practitioner. This data has been collected in the frame of the same research project as mentioned in footnote 5 .

7 At the end of the interview, the patient refuses to take part in an 'assertiveness training'. Her reason for this refusal is that she does not want to participate in a group. She does not challenge the usefulness of seeking remedy for her 'subassertiveness'.
} 
From these two examples, it can be concluded that (re)formulation of the patient's talk in the initial interview is done by means of the conversational formulation-decision pair. As shown above, this device is also used in the exploratory type of interviewing. But there is an important difference. In exploratory interviewing, the formulation-decision pair is not used for formulating the professional problem definition, because the professional problem definition is not formulated in the interview at all. This difference may be related to another difference between the two types of interviewing. On the one hand, predominantly collaborative interviews appear to be more or less purposefully and coherently integrated wholes, as if from beginning to end the patient and interviewer are cooperating in order to reach a common goal. On the other hand, exploratory interviews rather seem to be mere collections of disparate conversational fragments. In the exploratory style of interviewing, the relationship between the diverse parts of the conversation is not articulated in the interview itself; by contrast, in the collaborative interviews, parties appear to orient themselves explicitly to a cumulative understanding of such relationships. The two examples of the collaborative style of interviewing represented here are highly suggestive in this respect. In both cases, we see how an interviewer's second formulation explicitly builds upon a confirmation by the patient of a previous formulation.

\section{The patient's role in the formulation process}

It goes without saying that in interrogatory as well as in exploratory interviews, the patient does not play an active part in the process of constructing a professional problem definition. The patient's talk is merely an object of a professional interpretative work which is not disclosed in the interview itself. In contrast, in collaborative interviews, the professional formulation of the patient's talk depends upon the patient's active involvement in this process. Because formulations form adjacency pairs with decisions, the course of collaborative interviews depends upon the patient's decisions, i.e. on his confirmations and disconfirmations. This raises the question what it precisely is that the patient decides on. In order to answer this question, let us first look at what the patient typically does in a decision slot following an interviewer's formulation.

From an analytic point of view, the patient basically has three options: plain disconfirmation, plain confirmation, and qualified confirmation. According to the conversation analytic literature (in particular Pomerantz, 1984, and Heritage and Watson, 1979), plain disconfirmation is strongly dispreferred. Therefore, it is not a complete surprise that we did not find instances of it in our data. Plain confirmation, on the other hand, was not rare. A good example is the patient's response in Extract 7 to the interviewer's formulation "You are an introvert": "Yes, one hundred percent. [...] Yes, I am an introvert, yes". As already noted, here the patient's utterance cannot only be heard as confirming the interviewer's label, but also as endorsing that it is inferred from his talk in a proper way. In other words, the patient does confirm that the label 'introvert' is a valid designation of his behavior, as described in his talk and as correctly paraphrased by the interviewer. 
Similarly, in Extract 8, the patient's "Yes" can be heard as not only indicating a positive response to the immediately preceding question ("If you understand its purport?"), but at the same time, as confirming that the term 'subassertive' is a valid designation for the patient's "not daring to say no" - at least, in the interview it is taken as such. The diagnosis 'subassertive' functions in the following parts of the interview as a 'fact'. Thus, retrospectively, i.e. as an effect of the course of the interview, the patient appears to have agreed with the interviewer's treatment of the patient's "not daring to say no" as a sign of 'subassertiveness'. By comparing, however, the mere "Yes" of this patient with the other patient's elaborate confirmations ("Yes, one hundred percent. It keeps simmering in my stomach. Yes, I am an introvert, yes"), it becomes clear that there is a notable lack on this patient's part of showing an understanding of the purport of the interviewer's formulation. We infer from this absence that the patient's "Yes" cannot be taken as a true confirmation of the designation 'subassertive'. Rather, the patient abstains from disconfirming the interviewer's formulation, and thus allows the interviewer to proceed.

The next extract shows a similar lack of the patient's showing an understanding of the professional formulation:

\section{Extract $9^{8}$}

( $\mathrm{C}=$ counsellor; $\mathrm{P}=$ patient $)$

P1 Well, before Friday, I have to write an interim evaluation (at school) and one has to write down all the objectives that one wants to learn. And somehow, on the one hand, I think, well, I must not get too personal and, on the other hand, I must not come to be too concrete.

F C1 Yeah, yeah. You have a bit of a separation problem, isn't it?

D P2 Yes.

C2 How far must I go? Yeah, yeah.

P3 For, according to me, in the first half of the year I have gone too far.

In this extract, the patient confirms the professional formulation 'separation problem' (in P2), but does not expand upon it. In contrast, however, she takes up its non-professional paraphrase offered by the interviewer in the next turn ("how far must I go").

These examples (Extracts 8 and 9) show how the properties of the conversational formulation-decision pair enable the interviewer to present his professional assessments as shared ones. In particular, the dispreference assigned to disconfirmations allows the interviewer to proceed as if a confirmation had been offered. Remarkably, we have found this kind of non-disconfirmation almost only in cases in which the interviewer formulates a professional upshot, usually in diagnostic terms, of the patient's talk. It is much rarer in cases in which the interviewer formulates either the gist of what was said by the patient previously, or its upshot, in non-professional terms (as in Extract 7).

\footnotetext{
8 The client is a woman referred by a general practitioner. This data has been collected in the frame of the same research project as mentioned in footnote 5 .
} 
We conclude from this unequal distribution of qualified and plain confirmation that the formulation-decision pair does not have the same function in different stages of the construction process of the professional problem definition. In the first stages of the process, the formulation-decision pair appears to have the function of generating paraphrases, i.e. various descriptions of the patient's behavior and experiences upon which the patient and the interviewer sincerely attempt to agree. In the later stages, the pair rather appears as a means for the interviewer to inform the patient about his professional interpretation and, at the same time, to test whether or not the patient resists the label. This leads us to make a gross distinction between two types of formulation in the initial interview in psychotherapy:

(a) Formulations of the gist of the patient's talk. Although these formulations probably will be constrained by professional standards defining what counts as evidence for a professional problem definition, they nevertheless are not professional in a strict sense, but rather belong to the patient's discourse. These formulations typically are followed by qualified confirmations, specifying the conditions under which the interviewer's formulations are justified in the patient's view. When these formulations have been confirmed by the patient, they function as evidence for a second type of formulation:

(b) The formulation of the professional upshot. This is the truly professional formulation of the problems at hand; this kind of formulation typically is confirmed by the patient. Still, in many cases the confirmation is minimal, suggesting rather a nondisconfirmation of the professional judgment.

\section{Protoprofessionalization and its effects}

One of the main effects of the (re)formulation process in the initial interview in psychotherapy, viz., the formulation of the gist of the patient's talk and the subsequent professional upshot of that gist, is a certain kind of 'education' or 'socialization' of the patient into the professional discourse. The least important aspect of this 'education' is that the patient learns what the appropriate labels are (such as 'subassertive' and 'separation problem'). More important is that he learns which behaviors and experiences are considered relevant by therapists and, more specifically, how they are to be formulated in order to count as evidence for a specific professional designation. By being shown, already in the initial interview, how everyday biographical events can be translated into professional interpretations, the patient learns how to be a competent patient, i.e. how to present experiences competently. In this way, the patient becomes a proto-professional. ${ }^{9}$

\footnotetext{
9 The concept of 'protoprofessionalization' has been introduced by De Swaan as a general term for the processes of 'medicalization', 'psychologization' and 'juridization' of everyday life. It is the effect of the process of professionalization on the people outside of the professions. By the process of 'protoprofessionalization' people increasingly orient themselves in everyday life to the fundamental notions and stances of the professions. The degree of 'protoprofessionalization' which prevails in someone's personal network facilitates his or her access to professional services. See De Swaan (1990).
} 
Our data shows how protoprofessionalization, which is a well-known effect of psychotherapy, begins already in the initial interview. Another related phenomenon is also observed, namely that the interviewer tolerates a patient's display of professional competence, something which is rather uncommon in professional encounters. An example of this is presented in the next extract. The patient is a woman who had taken part in an 'assertiveness training' about two years ago.

\section{Extract $10^{10}$}

( $\mathrm{C}=$ counsellor; $\mathrm{P}=$ patient $)$

P1 But yet, I really have a very negative self-image, so uh, if something is going on, well, then again, I am thinking I am nowhere. I am the one who is doing it all wrong all the time.

$\mathrm{C} 1$ What causes you to think that you have such a negative self-image? What does this mean for you?

P2 Yes. The fact that, well, if something is going on, well, I am the one who is doing it wrong all the time, you know.

[data omitted]

C2 Suppose you would be asked to give a description of yourself. How would you describe yourself then?

P3 Well, in fact, like a little child. Not grown up. Or dependent. That is what I feel in fact.

C3 And what does that mean? Such a description that you give of yourself, well, as you say in fact like a little child and dependent, you are confronted with something that you perhaps do not like at all, isn't it? You would like to avoid the confrontation. Is that right?

P4 Yes, I think so. Because each time when I have to talk about myself I start crying. I cry a lot indeed, you know. I start crying very easily.

[data omitted]

It happened just recently, there was someone in our neighborhood, they got a baby. And we just talked for a second and then, very easily, I get tears in my eyes, or rather, tears come into my eyes.

C4 Why is it that you respond to things so emotionally? Was it a nice conversation, did you like the contact?

P5 Well uhh. I don't know, well, in the past when, well, I think at the time I was an adolescent or so. ((sobbing))

In his subsequent report, the counsellor referred to the patient as an 'insecure' and 'dependent' woman with a 'negative self-image' and a 'low emotional threshold'. The excerpt shows that the patient herself was formulating many of these more or less professional designations. As in the other extracts, it is the counsellor who articulates the relationship between the professional and the lay problem definitions. But

10 This extract consists of excerpts from an initial interview that was held by the same counsellor as in Extract 8 . The client is a woman referred by a general practitioner. 
whereas normally, the interviewer formulates the patient's prior utterances, here, by contrast, it is the patient who first gives the professional formulation. Thus, the interviewer does not formulate the patient's utterance, but rather takes it as a gloss to be unpacked. Doing this enables the interviewer to determine whether or not he can confirm the patient's formulation, whereas in the normal case the patient confirms (or rather does not disconfirm) the interviewer's formulations.

Compare the attitude of this interviewer vis-à-vis that of the doctor in the following extract.

Extract 11 (from Lacoste, 1981: 172)

( $\mathrm{D}=$ doctor; $\mathrm{P}=$ patient $)$

D1 How long have you had this pain in your stomach?

P1 I've never had a pain in my stomach, I have a pain in my spleen.

D2 Listen, the spleen you're not supposed to know where that is, you had a pain in the stomach.

P2 I have a pain there. ((designative gesture))

D3 What do you call that? That's the stomach. You have a pain in the stomach.

P3 If you say so.

In this extract, the doctor tells the patient that he does not expect her to engage in formulating candidate diagnoses. His "Listen, the spleen you're not supposed to know where that is" (D2) is an indication, both for the patient and for us analysts, that he is not willing to engage in a collaborative kind of interview in which the patient's statement ("I have a pain in my spleen") would have been taken as a candidate formulation of the patient's problem. His utterance also makes it clear, for that matter, that he is not willing to engage in an exploratory kind of interview either, in which he would have taken this 'pain in my spleen' as a description of a 'fact' in the patient's life that could be explored.

\section{Conclusion}

In this paper, we have described the function of the formulation-decision pair in three types of interviewing. The investigatory type of interviewing, which typically occurs in the medical interview, is characterized by the absence of formulations. In the exploratory type of interviewing, which typically occurs in the psychiatric interview, the interviewer uses the formulation-decision pair for exploring the patient's experiences. He does not, however, formulate his professional assessment in the interview. In the collaborative type, which typically is found in psychotherapy, the formulation-decision pair is the main interactional device used by the interviewer for translating the patient's troubles into a professional problem definition. This formulation process appears to be composed of two stages:

(a) the formulation of the gist of the patient's talk so far, typically followed by a qualified confirmation specifying the conditions under which the interviewer's 
formulation is justified. When such a formulation is confirmed by the patient, it functions as evidence for a second type of formulation:

(b) the formulation of the professional upshot, which typically is confirmed by the patient in a minimal way, suggesting a non-disconfirmation of the professional judgment rather than a true confirmation.

It is suggested that the patient's 'protoprofessionalization', which is characteristic for psychotherapy, already begins in the initial interview and that it is an effect of the interviewer's specific use of the formulation-decision device in the interview.

\section{References}

Davis, Kathy, 1984. Problem (re)formulation in psychotherapy. Konteksten no. 4, Rotterdam: Institute for Preventive and Social Psychiatry.

De Boer, Fijgje and Tony Hak, 1986. Besluitvorming in de acute psychiatrie [Decision-making in emergency psychiatry]. Konteksten no. 7, Rotterdam: Institute for Preventive and Social Psychiatry.

De Swaan, Abram, 1990. From troubles to problems. In: A. de Swaan, The management of normality, 99-108. London/New York: Routledge.

Garfinkel, Harold and Harvey Sacks, 1970. On formal structures of practical actions. In: J.C. McKinney and E.A. Tiryakian, eds., Theoretical sociology, 337-366. New York: Appleton-Century-Crofts.

Hak, Tony, 1989. Constructing a psychiatric case. In: B. Torode, ed., Text and talk as social practice, 72-92. Dordrecht: Foris.

Hak, Tony, 1992. Psychiatric records as transformations of other texts. In: G. Watson and R. Seiler, eds., Text in context: Contributions to ethnomethodology, 138-155. Newbury Park, CA: Sage Publications.

Hak, Tony and Fijgje de Boer, forthcoming. Professional interpretation of patient's talk in the initial interview. In: J. Siegfried, ed., Therapeutic and everyday discourse as behavior change, 341-364. Norwood, NJ: Ablex.

Heritage, John and Rodney Watson, 1979. Formulations as conversational objects. In: G. Psathas, ed., Everyday language: Studies in ethnomethodology, 123-162. New York: Irvington.

Lacoste, Michèle, 1981. The old woman and the doctor. Journal of Pragmatics 5: 169-180.

Mishler, Elliot, 1984. The discourse of medicine. Norwood, NJ: Ablex.

Pomerantz, Anita, 1984. Agreeing and disagreeing with assessments: Some features of preferred/dispreferred turn shapes. In: J.M. Atkinson and J. Heritage, eds., Structures of social action. 57-101. Cambridge: Cambridge University Press.

Weingarten, Rüdiger, 1990. Reformulieringen in der Gesprächs-psychotherapie. In: K. Ehlich, A. Koerler, A. Redder and R. Weingarten, eds., Medizinische und therapeutische Kommunikation. Diskursanalytische Untersuchungen, 228-240. Opladen: Westdeutscher Verlag. 